\title{
Rompiendo un mito en el control de la hemoglobina/hematocrito postransfusión
}

\section{Breaking a myth in postransfusion control of hemoglobin/hematocrit}

\author{
Juan Mauricio Pardo, Uriel Panqueva Martínez, \\ Ángela Fernanda EsPinOsa • Bogotá, D.C.
}

\section{Resumen}

Habitualmente se ha solicitado la hemoglobina/hematocrito después de las seis horas como parámetro para evaluar el resultado terapéutico de la transfusión sin que exista evidencia clara para hacerlo.

Objetivo: determinar la concordancia de los niveles de hemoglobina/hematocrito en los primeros 15 minutos de terminada la transfusión comparado con la toma de éstos a las seis horas en pacientes con anemia crónica sin sangrado activo, hemólisis o hiperesplenismo.

Materiales y métodos: se realizó un estudio, observacional, analítico de concordancia que analizó la consistencia entre dos mediciones de hemoglobina/hematocrito tomada dentro de los primeros 15 minutos de terminada la transfusión y seis horas después en 41 pacientes con anemia de origen médico, sin sangrado activo.

Resultados: se encontró concordancia significativa alta entre la hemoglobina a los 15 minutos y seis horas después de la transfusión ( $\mathrm{p}<0.0001$ ) con un coeficiente de correlación intraclase de 0.8793 (IC 95: 0.7817, 0.9349); igualmente se encontró concordancia significativa alta entre el hematocrito a los 15 minutos y a las seis horas después de la transfusión ( $\mathrm{p}<0.0001)$ con un coeficiente de correlación intraclase de 0.8456 (IC 95\%: 0.7301, 0.9144).

Conclusiones y discusión: aunque es una práctica habitual la toma de hemoglobina-hematocrito seis horas después de la transfusión, sin evidencia en la literatura que lo respalde, este estudio encontró que existe concordancia alta, significativa entre la toma de la hemoglobina/hematocrito 15 minutos y seis horas después de terminada la transfusión en pacientes con anemia de origen médico, lo que permite una toma inmediata, ahorrando tiempo en la decisión clínica (Acta Med Colomb 2010; 35: 2-7).

Palabras clave: transfusión sanguínea, evaluación, error en la evaluación terapéutica.

\section{Abastract}

Measurement of hemoglobin/hematocrit is usually carried out 6 hours after transfusion, as a parameter allowing the assessment of therapeutic result. However, there is no clear evidence to support this practice.

Objective: to determine the concordance of the levels of hemoglobin/hematocrit 15 minutes after termination of transfusion, with regard to those measured 6 hours after the procedure, in patients with chronic anemia and without active bleeding, hemolysis, or hypersplenism.

Materials and methods: an observational, analytic, concordance study was carried out in order to analyze the concordance between 2 measurements of hemoglobin/hematocrit: 15 minutes after transfusion and 6 hours after transfusion. This was done in 41 diagnosed with anemia of medical origin, without active bleeding.

Results: a highly significant concordance was found between hemoglobin at 15 minutes and 6 hours after transfusion ( $\mathrm{p}<0.0001$ ), with an intraclass correlation coefficient of 0.8793 (IC 95: 0.7817,0.9349); highly significant concordance was also found between hematocrit at 15 minutes and 6 hours after transfusion ( $\mathrm{p}<0.0001$ ), with an intraclass correlation coefficient of 0.8456 (IC 95\%: 0.7301, 0.9144).

Conclusions and discussion: although it is common practice to determine hemoglobin/hematocrit 6 hours after transfusion (without supporting evidence reported in the literature), this study found a

Dr. Juan Mauricio Pardo Oviedo: Médico Internista, Universidad del Rosario, Fundacion Cardioinfantil, Jefe de Educación Médica Mederi; Dr. Uriel Panqueva Martínez: Médico Internista, Universidad del Rosario, Fundación Cardioinfantil; Dra. Ángela Fernanda Espinosa Aranzales: Enfermera, Epidemióloga. Directora del Programa de Enfermería de la Universidad del Rosario. Bogotá, D.C.

Declaración de Conflictos de Interés: Ninguno

Correspondencia. Dr. Juan Mauricio Pardo Oviedo. Hospital Universitario Mayor, Piso 9. Calle 24 No 29-61. Tel: 5600520 ext 4923

E-mail: juan.pardo@urosario.edu.co Recibido: 05/VIII/09 Aceptado: 11/II/10 
high, significant correlation between measurements carried out at 15 minutes and 6 hours after transfusion in patients with anemia of medical origin. Immediate measurement saves time in clinical decisions (Acta Med Colombiana 2010; 35: 2-7).

Keywords: blood transfusion, monitoring, therapeutic misestimetion.

\section{Introducción}

La anemia está dentro de las patologías que con más frecuencia enfrenta el clínico; el diagnóstico de la misma se establece según la causa y la presentación del cuadro, pero el diagnóstico definitivo se hace de acuerdo con el nivel de hemoglobina y hematocrito. La transfusión de glóbulos rojos para el manejo de la anemia es una de las intervenciones terapéuticas que con más frecuencia se realiza; aproximadamente 81 millones de unidades de sangre son recolectadas cada año en el mundo (1) y se calcula que a nivel mundial la incidencia de transfusión de glóbulos rojos es de 44.7 a 50.1 unidades transfundidas por 1000 personas/ año (2). En los Estados Unidos 20 millones de componentes sanguíneos son transfundidos cada año en 4 millones de pacientes y en Colombia se transfunden anualmente unas 850.000 unidades de sangre y sus componentes de las cuales $50 \%-60 \%$ corresponden a glóbulos rojos (3).

Como una forma de evaluar el efecto terapéutico de todas estas transfusiones de glóbulos rojos se viene solicitando el control de hemoglobina y hematocrito en forma rutinaria después de seis horas postransfusión, práctica habitual y que se encuentra referenciada en algunos textos de hematología (4), pero a la cual las diferentes guías sobre terapéutica transfusional no hacen ninguna referencia (5-7, 9-14).

Teniendo en cuenta que los glóbulos rojos son corpúsculos que no se difunden fuera del espacio intravascular y al tener una osmolaridad similar a la del plasma tampoco van a producir ningún movimiento de agua o de electrolitos; al transfundir una unidad de glóbulos rojos a una velocidad de $1.25-2.50 \mathrm{ml} / \mathrm{min}$, esta pequeña cantidad de sangre que pasa minuto a minuto tardaría unos pocos minutos en alcanzar su concentración de equilibrio; es decir, que al final de la transfusión ya estaría cerca de lograr una concentración homogénea en la hemoglobina y hematocrito $(15,16)$.

Hay sólo dos trabajos en la literatura, los cuales no son de concordancia y que evaluaron el tiempo óptimo al que se debe tomar el control de hemoglobina y hematocrito que sea representativo del control final de estos parámetros $(17,18)$; nosotros hemos pensado que no hay razones fisiológicas, ni evidencia suficiente en la literatura que sustenten el tiempo habitual para la realización de una medida tan frecuente como es el control de hemoglobina/hematocrito por lo cual proponemos en nuestro trabajo un cambio en la práctica médica con la toma más temprana de estos parámetros.

\section{Métodos}

Se realizó un estudio prospectivo observacional, analítico, de concordancia, que analizó la consistencia entre dos mediciones de hemoglobina en 41 pacientes que requirieron transfusión de glóbulos rojos en pacientes con anemia de origen médico hospitalizados en la Fundación Cardioinfantil.

Los siguientes fueron los criterios de inclusión: 1) pacientes mayores de 18 años, 2) presencia de anemia de causa médica sin evidencia de sangrado activo en las últimas 24 horas siendo los criterios de exclusión: 1) pacientes con algún tipo de discapacidad que le impidiera entender o firmar el consentimiento informado en ausencia de representante legal,2) patologías médicas con esplenomegalia, 3) anemias hemolíticas, 4) necesidad de coloides o cristaloides en las últimas 24 horas mayores a los requerimientos basales, 5) uso de diuréticos.

La cifra de hemoglobina pretransfusión se tomó de la historia clínica y se evaluaron dos nuevas muestras después de la transfusión; una a los quince minutos y otra después de seis horas. La muestra de sangre se realizó mediante punción y la medición se efectuó en un equipo fotómetro Hemocue, el cual es un hemoglobinometro portátil que utiliza el método de la azida-metahemoglobina. El método se basa en la hemolisis completa de la muestra de sangre por analizar mediante sodio-deoxicolato, con mediciones realizadas en dos longitudes de onda $(570 \mathrm{~nm}$ y $880 \mathrm{~nm})$ proporcionando resultados en $15 \mathrm{~s}-45 \mathrm{~s}$, con una buena correlación con otros métodos de medición y en diferentes situaciones clínicas (19-21). El hematocrito se calculó a partir de los resultados de hemoglobina multiplicando este último resultado por tres (22). El estudio fue aprobado por el Comité de Ética de la Institución y el consentimiento informado fue obtenido de todos los pacientes. El tamaño de la muestra fue de 41 pacientes el cual fue obtenido mediante la ecuación de tamaño de muestra de Streiner y Norman.

Se evaluó la normalidad de la hemoglobina y hematocrito a los 15 minutos y 6 horas mediante la prueba de Kolmogorov-Smirnov (KS), a un nivel de significancia del $5 \%(\mathrm{p}<0.05)$.

Para evaluar la concordancia entre hemoglobina a los 15 minutos y a las 6 horas y hematocrito a los 15 minutos y 6 horas, se utilizó el coeficiente de correlación intraclase CCI y con un intervalo de confianza del $95 \%$ y se evaluó la concordancia a un nivel de significancia del 5\% ( $<<0.05)$. Se tomó como criterio de clasificación de concordancia con el CCI: < 0.40 baja concordancia, entre $0.40-0.59$ moderada concordancia, entre 0.60-0.79 medianamente alta concordancia y 0.80-1.0 alta concordancia.

La sistematización de la información se realizó en paquete SPSS para Windows versión 15.0.

\section{Resultados}

El grupo de estudio lo conformaron 41 pacientes que requirieron transfusión por anemia, en un rango de edad 
entre los 21 años y 99 años (Tabla 1) con un promedio y una desviación estándar de 57.6 años y de \pm 18.9 años respectivamente, con un rango y donde el grupo con mayor frecuencia fue de 70 años a 79 años y por género mostró un distribución muy similar en hombres un $53.7 \%(\mathrm{n}=22)$ y mujeres un $46.3 \%(n=19)$. El promedio del índice de masa corporal del grupo fue de $24.4 \pm 5.4$, el mínimo fue de 17.18 y el máximo de 42.96 y se encontró sobrepeso en un $26.2 \%$ de los pacientes (Tabla 2).

Dentro de las patologías asociadas a la anemia que fueron la indicación de la transfusión, se encontró una mayor frecuencia de las enfermedades neoplásicas en un 58.5\% predominando las de origen hematopoyético (Tabla 3 ).

En el grupo de pacientes se encontró en un $95.1 \%$ (39 pacientes) se trasfundieron con más de una unidad, siendo lo más frecuente con dos unidades (75.6\%), con tres unidades un $17.1 \%$ y con cuatro en un solo paciente.

\section{Concordancia}

Se encontró concordancia significativa alta entre la hemoglobina a los 15 minutos y 6 horas después de la transfusión $(\mathrm{p}<0.0001)$ con un coeficiente de correlación intraclase de 0.8793 (IC 95: 0.7817, 0.9349) (Figura 1).

También se encontró concordancia significativa alta entre el hematocrito a los 15 minutos y a las 6 horas después de la transfusión $(\mathrm{p}<0.0001)$ con un coeficiente de correlación intraclase de 0.8456 (IC 95\%: 0.7301, 0.9144) (Figura 2).

Mostró normalidad la distribución de la hemoglobina a los 15 minutos ( $\mathrm{p}=0.200, \mathrm{KS})$, a las 6 horas $(\mathrm{p}=0.200$, $\mathrm{KS}$ ), de la misma forma el hematocrito a los 15 minutos $(\mathrm{p}=0.108, \mathrm{KS})$ y a las 6 horas $(\mathrm{p}=0.200, \mathrm{KS})$.

Las medidas descriptivas de la hemoglobina y hematocrito pre y postranfusión a los 15 minutos y 6 horas se presenta en la Tabla 4.

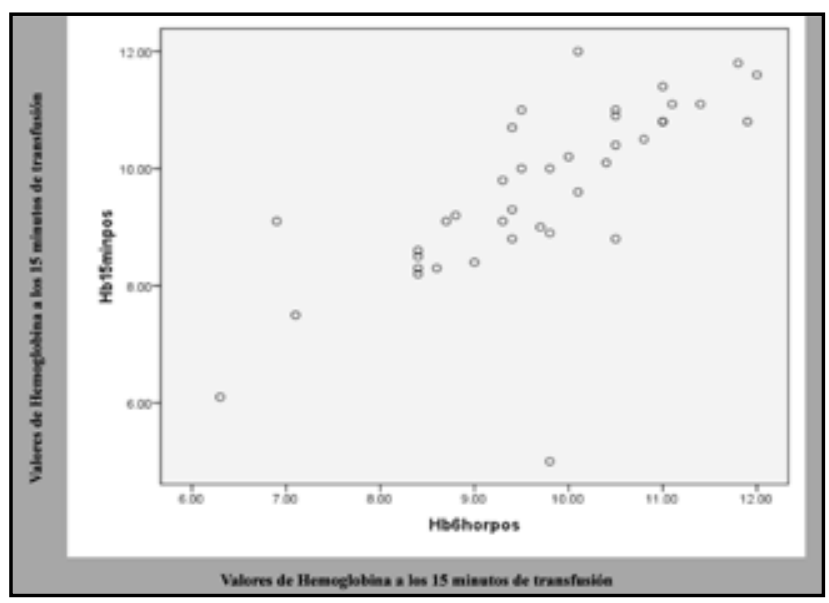

Figura 1. Diagrama de concordancia de valores de hemoglobina a los 15 minutos y 6 horas de pacientes que requirieron transfusión por anemia, Fundación Cardioinfantil, Bogotá D.C. 2005-2007.
Tabla 1. Distribución por grupos etáreos de los pacientes que requirieron transfusión por anemia, Fundación Cardioinfantil, Bogotá D.C. 2005-2007.

\begin{tabular}{|ccc|}
\hline Grupos Etáreos & Fc x min & Porcentaje(\%) \\
\hline $20-29$ & 2 & 4.9 \\
$30-39$ & 7 & 17.1 \\
$40-49$ & 4 & 9.8 \\
$50-59$ & 8 & 19.5 \\
$60-69$ & 8 & 19.5 \\
$70-79$ & 9 & 22.0 \\
$>=80$ & 3 & 7.3 \\
\hline TOTAL & $\mathbf{4 1}$ & $\mathbf{1 0 0 . 0}$ \\
\hline Fc: Frecuencia cardíaca & & \\
\hline
\end{tabular}

Tabla 2. Distribución del índice de masa corporal en los pacientes que requirieron trans fusión por anemia, Fundación Cardioinfantil, Bogotá D.C. 2005-2007.

\begin{tabular}{|ccc|}
\hline Índice de masa corporal & Fc $\mathbf{x}$ min & Porcentaje(\%) \\
\hline$<20$ & 6 & 14.6 \\
$20-24.9$ & 20 & 48.8 \\
$25-29.9$ & 10 & 24.4 \\
$>=30$ & 5 & 12.2 \\
\hline Total & $\mathbf{4 1}$ & $\mathbf{1 0 0 . 0}$ \\
\hline Fc: Frecuencia cardíaca & & \\
\hline
\end{tabular}

Tabla 3. Distribución de diagnósticos asociados a transfusión de los pacientes que requirieron transfusión por anemia, Fundación Cardioinfantil, Bogotá D.C. 2005-2007.

\begin{tabular}{|lcc|}
\hline Variable & Fc x min & Porcentaje (\%) \\
\hline Leucemias & 11 & 26.8 \\
Linfomas & 8 & 19.5 \\
Otros cánceres & 5 & 12.2 \\
Infecciones & 2 & 4.9 \\
Otras enfermedades médicas & 12 & 29.3 \\
Sangrados & 3 & 7.3 \\
\hline Total & $\mathbf{4 1}$ & $\mathbf{1 0 0 . 0}$ \\
\hline Fc: Frecuencia cardíaca & & \\
\hline
\end{tabular}

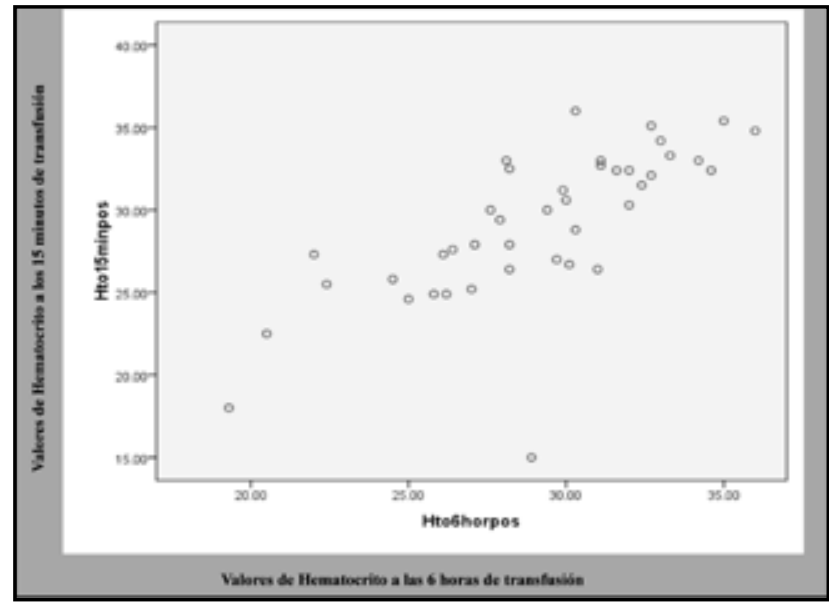

Figura 2. Diagrama de concordancia de valores de hematocrito a los 15 minutos y 6 horas de pacientes que requirieron transfusión por anemia, Fundación Cardioinfantil, Bogotá D.C. 2005-2007. 
Tabla 4. Medidas descriptivas de la hemoglobina (Hb) y hematocrito (HTo), pretransfusión, a los 15 minutos y 6 horas postransfusión, de pacientes que requirieron transfusión por anemia, Fundación Cardioinfantil, Bogotá D.C. 2005-2007.

\begin{tabular}{|c|c|c|c|c|c|c|}
\hline & Hb Pre & Hb Pos 15 minutos & Hb Pos 6 horas & HTo Pre & HTo Pos 15 Minutos & HTo Pos 6 horas \\
\hline $\mathrm{N}$ & 41 & 41 & 41 & 41 & 41 & 41 \\
\hline Media & 7.675 & 9.954 & 98.667 & 23.251 & 29.871 & 29.437 \\
\hline Mediana & 7.800 & 10.100 & 98.000 & 23.400 & 30.300 & 30.000 \\
\hline Moda & 7.8 & 10.8 (a) & 8.40 (a) & 24.0 & 32.4 (a) & 28.2 (a) \\
\hline Desv. Típ. & 10.774 & $13 . .824$ & 129.581 & 31.419 & 41.601 & 38.031 \\
\hline Varianza & 11.609 & 19.110 & 167.912 & 98.716 & 173.066 & 144.639 \\
\hline Asimetría & -.494 & -.487 & -.504 & -.938 & -.533 & -.695 \\
\hline Error Típ. de asimetría & 369 & 369 & 378 & 369 & 369 & 369 \\
\hline Rango & 6.1 & 5.9 & 5.70 & 16.7 & 18.0 & 16.7 \\
\hline Mínimo & 4.4 & 6.1 & 6.30 & 13.2 & 18.0 & 19.3 \\
\hline Máximo & 10.5 & 12.0 & 12.00 & 299 & 36.0 & 36.0 \\
\hline
\end{tabular}

\section{Discusión}

Nuestros resultados son similares a los encontrados previamente por Wiesen et al, (17). quienes ya en su trabajo de 1994 en 52 pacientes con anemia de origen medico, sin sangrado activo tomando controles de hemoglobina a los 15 minutos y 24 horas después de la transfusión, no hallaron diferencias significativas en ninguno de estos controles, igualmente este estudio menciona que no existía al momento literatura que respaldara el procedimiento habitual. En 1997 en el trabajo de Elizalde (18), en 35 pacientes con hemorragia de vías digestivas altas sin sangrado activo pero tomando muestras en diferentes tiempos durante un periodo de 24 horas encontraron también que no hubo diferencias significativas entre los distintos tiempos de tomas del control de hemoglobina. Estos resultados son similares a los escasos estudios realizados en niños; donde las mediciones en 24 infantes a los 15 minutos y 6 horas después encontraron resultados idénticos en las dos muestras.

Estos hallazgos son corroborados por este estudio, pero con el valor agregado de que es el primer trabajo en la literatura de concordancia y en el cual se encuentra una concordancia significativa alta entre el control de la hemoglobina/hematocrito tomados a los 15 minutos de terminada la transfusión y el obtenido seis horas después en pacientes sin sangrado activo, hemodinámicamente estables, sin hemólisis, ni esplenomegalia y contradice la práctica habitual de tomar estos controles seis u ocho horas después sin que exista ninguna evidencia en la literatura que la respalde.

La limitación de nuestro estudio es que es aplicable sólo a los pacientes transfundidos por patología médica sin inestabilidad hemodinámica, pero que corresponden al 40\%-50\% aproximadamente de los pacientes que son transfundidos en el mundo, aunque los hallazgos sugerirían poder aplicarlo en otro tipo de pacientes.

La toma precoz del control de hemoglobina/hematocrito permitirá ahorro de tiempo, recursos y toma de decisiones más rápidas con beneficio para el paciente, haciendo los servicios más eficientes, basados no en la tradición, sino en protocolos con respaldo en la evidencia, con conclusiones prácticas y reales.

Consideramos que es necesario realizar nuevas mediciones en otras patologías que requieran transfusión para mirar la concordancia en otros escenarios.

\section{Referencias}

1. Organización mundial de la salud. 10 datos sobre la transfusion de sangre. (en linea) www.who.int/features/factfiles/blood_transfusion/es/. (Consultado 20 enero 2010).

2. Cobain TJ, Vamvakas EC, Wells A, Titlestad K. A survey of the demographics of blood use. Transfus Med 2007; 17: 1-15.

3. Villegas N. Transfusiones, Indicaciones para el Uso de la Sangre y Componentes Sanguíneos. En Guías para el Manejo en Urgencias, Fundación Santa Fe de Bogotá. 2006.p.1026 - 32.

4. Hoffman R, Edward J. Benz Jr. Principles of red blood cell transfusion. En Hematology: Basic Principles and Practice, Churchill Livingstone, editors. 4 ed. Philadelphia, Pennsylvania; 2005.p. 2423-30

5. Murphy MF, Wallington TB , Kelsey P, Boulton F, Bruce M, Cohen H, et al. Guidelines for the clinical use of red cell transfusions. Br J Haematol 2001; 113: 24-31.

6. Goodnough LT, Brecher ME, Kanter MH,AuBuchon JP. Transfusion Medicine. First of two parts - blood transfusion. N Engl J Med 1999; 340: 438-47.

7. Salazar M. Guidelines for the transfusion of blood and its components. Pan Am J Public Health 2003; 13: 183-90.

8. Simpon TL, Alverson DC, AuBuchon J, Cooper ES, DeChristopher D, Glenn GC, et al. Practice parameter for the use of red blood cell transfusions. En Red Blood Cell Administration Practice Guideline Development Task Force of the College of American Pathologists. Arch Pathol Lab Med 1998; 122: 130-8.

9. American Society of Anesthesiologists Task Force on Perioperative Blood Transfusion and Adjuvant Therapies. Practice guidelines for perioperative blood transfusion and adjuvant therapies: an updated report by the American Society of Anesthesiologists Task Force on Perioperative Blood Transfusion and Adjuvant Therapies. Anesthesiology 2006; 105: 198-208.

10. American Society of Anesthesiologists Task Force on Blood Component Therapy. Practice guidelines for blood therapy. Anesthesiology 1996; 84: 732-47.

11. Stehling L, Luban NL, Anderson KC, Sayers MH, Long A, Attar S, et al. Guidelines for blood utilization review. Tranfusion 1994; 34: 438-48.

12. Cable R, Carlson B, Chambers L, Kolins J, Murphi S, Tilzer L,et al. Practice guidelines for blood transfusion: A compilation from recent peer reviewed literature, American Red Cross Publication 1416, May 2002.

13. Heading R, Campbell G, Campbell N, Cooper T, Creighton D, Cutler F. NHS Quality Improvement Scotland. Clinical Standards: Blood Transfusion. NHS. Edinburgo Sept 2006: 1-42.

14. Lichtman A, Beutler E, Kipps T, Seligsohn U, Kaushansky K, Prchal T. Preservation and Clinical Use of Erythrocytes and Whole Blood. En: Williams Hematology. 7 ed; New York; McGraw-Hill Companies; 2005.p.131.

15. Lagora M, Castella D, Castrillo A, Cid J, Contreras E, Corteza A. Guía sobre 
la transfusión de componentes sanguíneos y derivados plasmáticos, Sociedad Española de Transfusión Sanguínea, 3 edición. Madrid 2006.p.2-47

16. Fullerton GD, Kanal KM, Cameron IL. On the osmotically unresponsive water compartment in cells. Cell Biol Int 2006; 30: 74-7.

17. Wiesen AR, Hospenthal DR, Byrd JC, Glass KL, Howard RS, Diehl LF. Equilibration of hemoglobin concentration after transfusion in medical inpatients not actively bleeding. Annals Intern Med 1994; 121: 278-80.

18. Elizalde JI, Clemente J, Marín JL, Panés J, Aragón B, Mas A, et al. Early changes in hemoglobin and hematocrit levels after packed red cell transfusion in patients with acute anemia. Transfusion 1997; 37: 573-6.

19. Gómez-Escolar Viejo L, Sala GS, Azorin JM, Laudemia R, Sánchez J, Re- gadera MP. Reliability of hemoglobin measurement by HemoCue in patients with gastrointestinal bleeding. Gastroenterol Hepatol 2009; 32: 334-8.

20. Richards NA, Boyce H, Yentis SM. Estimation of blood haemoglobin concentration using the HemoCue during caesarean section: the effect of sampling site. Int J Obstet Anesth 2010; 19: 67-70.

21. Munoz E. Naveira A, Romero R, Ramirez R. Exactitud y precisión de la determinación inmediata de hemoglobina con el HemoCue B Hemoglobin en pacientes urgentes, quirúrgicos y críticos. Rev Esp Anestesiol Reanim 2003; 50: 332-9.

22. Stine-Marti AE, Lotsperch-Steininger CA, Koepke JA. Clinical Haematology: Principles, procedures, correlation. 2 ed.; New York: Lippincot; 1998.p.113 